\title{
A new use for an old index: preoperative high-density lipoprotein predicts recurrence in patients with hepatocellular carcinoma after curative resections
}

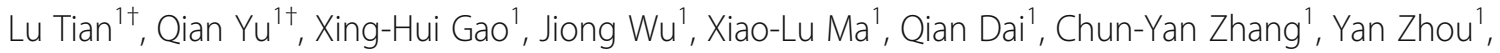
Yi-Chi Zhang ${ }^{1}$, Bai-Shen Pan ${ }^{1}$, Jian Zhou ${ }^{2}$, Jia Fan², Xin-Rong Yang ${ }^{2^{*}}$ and Wei Guo ${ }^{1,2^{*}}$

\begin{abstract}
Background: Hepatocellular carcinoma has high incidence and mortality worldwide. Liver is the site of most metabolic biotransformation, which could reflect the status of cells. Most plasma apolipoproteins, endogenous lipids and lipoproteins are synthesized in the liver. Therefore, the effects of lipid metabolites on prognosis of HCC deserved to be explored.

Methods: We prospectively included 58 healthy donors (HD), 50 chronic hepatitis $(\mathrm{CH})$ patients and a training cohort of 189 patients with HCC who underwent curative resections at Zhongshan Hospital from January 2012 to August 2012. We identified the optimal HDLpo cutoff value at $0.98 \mathrm{mmol} / \mathrm{L}$ and used it to stratify patients into lowor high-HDLpo groups for the entire cohort and four low-recurrent-risk subgroups. We also included an independent validation group of 182 HCC patients to validate this cutoff value. Prognostic values of HDLpo and other factors were determined by Kaplan-Meier curves and the Cox proportional hazards model.

Results: The low-HDLpo group had a higher median tumor grade $(P=0.020)$ and a higher recurrence rate $(P=0$. 032). Results of multivariate analysis showed that preoperative $\gamma$-glutamyl transpeptidase (GGT) and HDLpo were independent predictors of recurrence. Moreover, the predictive value of HDLpo was retained in four low-recurrentrisk subgroups. As expected, clinicopathologic characteristics and predictive values were similar in the validation and training cohorts.
\end{abstract}

Conclusions: $\mathrm{HDL}_{\mathrm{PO}}$ is an accessible predictor of HCC recurrence after liver resections that can help identify patients who need more careful monitoring and follow-up care.

Keywords: HDL, HCC, Lipid metabolites, Prognosis

\footnotetext{
*Correspondence: yxr_2@163.com; guo.wei@zs-hospital.sh.cn

${ }^{\dagger}$ Equal contributors

Department of Liver Surgery, Liver Cancer Institute, Zhongshan hospital,

Fudan University, Key Laboratory of Carcinogenesis and Cancer Invasion,

Ministry of Education, 136 Yi Xue Yuan Road, Shanghai 200032, People's

Republic of China

'Department of Laboratory Medicine, Zhongshan Hospital, Fudan University,

136 Yi Xue Yuan Road, Shanghai 200032, People's Republic of China
} 


\section{Background}

Hepatocellular carcinoma (HCC) has third highest cancer mortality worldwide $[1,2]$. Although advances in treatment have improved survival of patients with HCC, they suffer high post-surgical recurrence and metastasis rates [1, 3]. Despite the common use of alfa-fetoprotein (AFP) to diagnose and predict recurrence of $\mathrm{HCC}$, its positive predictive rate is about $70 \%$ [4]. Therefore, identifying reliable indicators for patients with a high risk of post-surgical relapse is imperative to provide patients with optimal adjuvant therapy.

Metabolites are the best molecular indicators of cell status, and liver is the site of most metabolic biotransformation [5]. Most plasma apolipoproteins, endogenous lipids and lipoproteins are synthesized in the liver [6, 7]. Although high-density lipoprotein (HDL), as a lipid metabolite, is known as "good cholesterol," with the main function of reverse cholesterol transport (RCT) and negative correlation with atherosclerosis [8], little is known about alterations of metabolism, especially HDL, and how these multi-level variations affect aggressive diseases and poor outcomes. Patients with hepatitis B or hepatitis $C$ have been shown to have possible lipid disorders, including decreased plasma HDL [5, 9]. However, whether HDL levels are correlated with HCC prognosis is unclear. We therefore designed this single-center prospective study of 189 patients in the training group and 182 patients in the validation group, as well as $58 \mathrm{HD}$ and $50 \mathrm{CH}$ patients, to explore whether HDL levels could predict $\mathrm{HCC}$ recurrence.

\section{Methods}

\section{Patients and specimens}

We included a training cohort of 189 patients and a validation cohort of 182 patients who had undergone curative resections at our hospital from January 2012 to September 2013 for HCC, but had not yet accepted any radiotherapy or chemotherapy before then. The method of diagnosing $\mathrm{HCC}$ in these patients, and the inclusion and exclusion criteria, were described in a previous study [10]. We have added the inclusion and exclusion criteria in the Method. The inclusion criteria were: (1) definitive pathological diagnosis of HCC based on World Health Organization criteria, (2) curative resection, defined as complete macroscopic removal of the tumor, and (3) no prior anticancer treatment; the exclusion criteria were: (1) with other malignant tumors before operation; (2) with preoperative infection; (3) with blood and immune system diseases. Peripheral venous blood samples were collected before surgery, from which serum was separated by centrifuge and saved in a $-80{ }^{\circ} \mathrm{C}$ refrigerator. Tumor differentiation was graded by the Edmondson system, and staging was determined by the Barcelona Clinic Liver Cancer (BCLC) classifications [11]. The Zhongshan Hospital Research
Ethics Committee approved this study, and all patients granted written informed consent.

\section{Follow-up}

Patients' postoperative surveillance included routine clinical and laboratory examinations, and imaging methods conducted within 1-3 months after their surgeries, to detect metastasis or recurrence [12]. Patients' baseline clinical characteristics were noted, including sex, age, tumor number, size, encapsulation and grade, satellite lesions, vascular invasion, ChildPugh grade, BCLC stage, AFP, alanine aminotransferase (ALT), $\gamma$-glutamyl transpeptidase (GGT), hepatitis virus $B$ antigen (HBsAg), HDL, and any recurrences. All recipients were followed regularly until recurrence, death, or termination of the study. Time to recurrence (TTR) was defined as the period between surgery and recurrence.

\section{RNA isolation and RCR}

Total RNA was isolated using TRIzol $^{\circledR}$ LS reagent (Invitrogen) according to manufacturer's instructions. The final elution volume ranged from 20 to $50 \mu \mathrm{l}$. The amount of specific transcripts was measured by RT-PCR using the 7500 quantitative Real-time PCR Machine.

\section{Statistical analysis}

Statistical analysis was used SPSS for Windows, Version 16.0 (SPSS Inc., Chicago, IL, USA). Student $t$ test, Pearson's $X^{2}$ test, and Fisher's exact test were used to compare differences between two groups. Univariate and multivariate analyses used the Cox proportional hazards regression model. The KaplanMeier method was used to generate survival curves, and differences between groups were assessed using the log-rank test. A two-sided $P$ value $<0.05$ was considered significant. The X-tile 3.6.1 software (Yale University, New Haven, CT) was used for bioinformatic analysis of the cohort data to determine the cutoff value of preoperative $\mathrm{HDL}\left(\mathrm{HDL}_{\mathrm{PO}}\right)$ for tumor recurrence [13].

\section{Results}

Lipid metabolism screening and optimal HDL

We examined four products of lipid metabolism, including total cholesterol (TC), triglycerides (TG), HDL, and low-density lipoprotein (LDL) in $189 \mathrm{HCC}$ patients before surgery, 58 healthy donors (HD) and 50 chronic hepatitis $(\mathrm{CH})$ patients. As expected, $\mathrm{HDL}_{\mathrm{PO}}$ was significantly lower in $\mathrm{HCC}$ patients than $\mathrm{CH}$ patients $(P<0.001)$ and HD $(P=0.0002)$, whereas TG was significantly lower than $\mathrm{CH}$ patients $(P=0.0094)$ and 

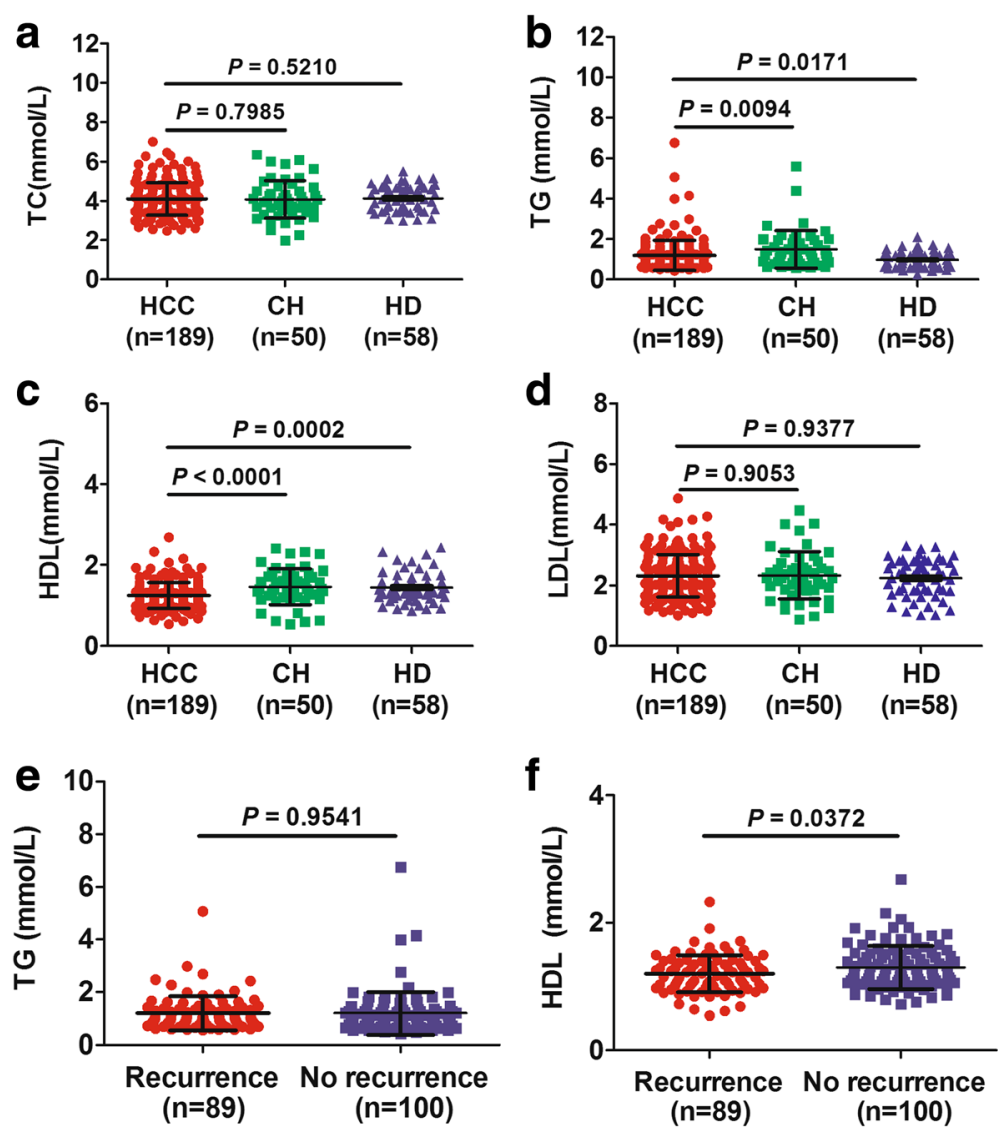

Fig. 1 Lipid metabolites in different groups. Levels of total cholesterol (TC; a), triglycerides (TG; b), high-density lipoprotein (HDL; $\mathbf{c}$ ) and low-density lipoprotein (LDL; d) in HCC patients,chronic hepatitis (CH) patients, and healthy donors (HD); and TG (e) as well as HDL (f) were analyzed in patients who suffered recurrences and those whose HCC did not recur

higher than HD $(P=0.0171$; Fig. 1 a-d). We then focused our research on these two markers to explore their differences in groups of patients who did, and who did not, suffer recurrences. We found only HDLPO clearly discriminated between the two groups $(P=0.037$; Fig. 1 e-f).

Results from X-Tile analysis revealed an optimal cutoff point for $\mathrm{HDL}_{\mathrm{PO}}$ at $0.98 \mathrm{mmol} / \mathrm{L}$ among the training cohort patients (Additional file 1: Figure S1), who were then stratified into those with $\leq 0.98 \mathrm{mmol} / \mathrm{L}$ (low $\mathrm{HDL}_{\mathrm{PO}}$ ) or $>0.98 \mathrm{mmol} / \mathrm{L}$ (high $\mathrm{HDL}_{\mathrm{PO}}$ ) for all subsequent analyses. The cutoff point generated by X-tile was then verified in an independent validation cohort to extend the universal use of $0.98 \mathrm{mmol} / \mathrm{L}$.

\section{Correlations between clinicopathologic characteristics and $\mathrm{HDL}_{\mathrm{PO}}$ levels in the training cohort}

The 189 patients included 162 men (85.71\%) and 27 women $(14.29 \%)$ with a median age of 54.4 years (range: 37-87 years). We found 166 (87.83\%) to be HBsAgpositive. The recurrence rate for all patients was $47.62 \%$ by the end of the follow-up (median TTR: 30.9 months; range:
0.6-37 months). After applying the $0.98 \mathrm{mmol} / \mathrm{L} \mathrm{HDL}_{\mathrm{PO}}$ cutoff, 32 patients $(16.93 \%)$ were in the low-HDL HO $_{\mathrm{PO}}$ group and 157 (83.07\%) in the high-HDL $\mathrm{HO}_{\mathrm{PO}}$ group.

Clinicopathologic characteristics included sex, age, tumor number and size, encapsulation and grade, satellite lesions, vascular invasion, Child-Pugh grade, BCLC stage, AFP, ALT, GGT, HBsAg, and recurrence. The low-HDL $\mathrm{HO}_{\mathrm{PO}}$ group had more advanced median tumor grades $(P=0.020)$ and a higher recurrence rate $(P=0.032$, Table 1$)$.

The prognostic value of $\mathrm{HDL}_{\mathrm{PO}}$ level for HCC patients in the training cohort

The Cox proportional hazard model was used to determine prognostic indicators. In univariate analysis, discriminating variables were preoperative GGT (hazard ratio [HR]: $1.004 ; 95 \%$ confidence index $[95 \% \mathrm{CI}]$ : $1.002-1.005 ; P=0.001)$ and $\mathrm{HDL}_{\mathrm{PO}}(\mathrm{HR}: 0.526 ; 95 \%$ CI: $0.322-0.858 ; P=0.010$ ). These two variables were further evaluated in multivariate analysis, which showed both GGT (HR, 1.986; 95\% CI, 1.308-3.017; $P=0.001)$ and $\mathrm{HDL}_{\mathrm{PO}}$ (HR: 0.519; 95\% CI: $0.318-$ 
Table 1 The correlation between clinicopathologic characteristics and HDLPO in the training cohort

\begin{tabular}{|c|c|c|c|c|c|}
\hline & & Number $(n=189)$ & Low-HDLPO group $(n=32)$ & High-HDLpo group $(n=157)$ & $P$ value \\
\hline \multirow[t]{2}{*}{ Sex } & Male & 163 & 28 & 135 & $0.821^{a}$ \\
\hline & Female & 26 & 4 & 22 & \\
\hline \multirow[t]{2}{*}{ Age } & $\leq 50$ & 69 & 13 & 56 & 0.596 \\
\hline & $>50$ & 120 & 19 & 101 & \\
\hline \multirow[t]{2}{*}{ Tumor number } & Single & 151 & 26 & 125 & 0.833 \\
\hline & Multiple & 38 & 6 & 32 & \\
\hline \multirow[t]{2}{*}{ Tumor size } & $\leq 5$ & 120 & 18 & 102 & 0.351 \\
\hline & $>5$ & 69 & 14 & 55 & \\
\hline \multirow[t]{2}{*}{ Tumor encapsulation } & None & 115 & 17 & 98 & 0.326 \\
\hline & Complete & 74 & 15 & 59 & \\
\hline \multirow[t]{2}{*}{ Satellite lesions } & No & 166 & 27 & 139 & $0.524^{a}$ \\
\hline & Yes & 23 & 5 & 18 & \\
\hline \multirow[t]{2}{*}{ Vascular invasion } & No & 118 & 17 & 101 & 0.321 \\
\hline & Yes & 71 & 15 & 56 & \\
\hline \multirow[t]{2}{*}{ Tumor grade } & $|-| \mid$ & 117 & 14 & 103 & 0.020 \\
\hline & III-IV & 72 & 18 & 54 & \\
\hline \multirow[t]{2}{*}{ Child-Pugh grade } & A & 178 & 29 & 149 & $0.347^{\mathrm{a}}$ \\
\hline & B & 11 & 3 & 8 & \\
\hline \multirow[t]{2}{*}{ BCLC stage } & $0+A$ & 138 & 24 & 114 & 0.781 \\
\hline & $B+C$ & 51 & 8 & 43 & \\
\hline \multirow[t]{2}{*}{ AFP, $\mu g / L$} & $\leq 400$ & 139 & 21 & 118 & 0.265 \\
\hline & $>400$ & 50 & 11 & 39 & \\
\hline \multirow[t]{2}{*}{$A L T, \mu g / L$} & $\leq 40$ & 178 & 32 & 146 & $0.124^{\mathrm{a}}$ \\
\hline & $>40$ & 11 & 0 & 11 & \\
\hline \multirow[t]{2}{*}{ GGT,IU/L } & $\leq 54$ & 110 & 17 & 93 & 0.559 \\
\hline & $>54$ & 79 & 15 & 64 & \\
\hline \multirow[t]{2}{*}{$\mathrm{HbsAg}$} & Negative & 23 & 1 & 22 & $0.100^{\mathrm{a}}$ \\
\hline & Positive & 166 & 31 & 135 & \\
\hline \multirow[t]{2}{*}{ Recurrence } & No & 100 & 11 & 89 & 0.032 \\
\hline & Yes & 89 & 21 & 68 & \\
\hline
\end{tabular}

Abbreviations: AFP, a-fetoprotein; ALT, alanine aminotransferase; GGT, Y-glutamyl transpeptadase; HBsAg, hepatitis B surface antigen; BCLC, Barcelona Clinic Liver Cancer ${ }^{\text {a}}$ Fisher's exact test

0.848; $P=0.009)$ to be independent prognostic risk factors of HCC recurrence (Table 2).

In the training cohort, Kaplan-Meier analysis showed the low- and high-HDL $\mathrm{PO}$ groups to significantly differ in median TTR (low-HDL $\mathrm{HO}_{\mathrm{PO}}$ group: 13.5 months; high-HDL $L_{\mathrm{PO}}$ group: 31.1 months; $P=0.0086$ Fig. 2a). The low-HDL ${ }_{\mathrm{PO}}$ group had a significantly higher recurrence rate $(65.63 \%)$ than the high-HDL ${ }_{P O}$ group (43.31\%). However, there were no significant statistics difference in OS between the two groups $(P=0.4880$ Fig. $2 \mathrm{c})$.

Further analysis in HCC patients with AFP negative or other low recurrent risks among the training cohort, we found that $\mathrm{HDL}_{\mathrm{PO}}$ level retained significant prognostic value in patients who were AFP-negative $(P=0.001)$, with no satellite lesions $(P=0.046)$, with complete encapsulated tumors $(P=0.001)$, and with BCLC stage $0+\mathrm{A}$ disease $(P=0.004$; Fig. 3 a-e). The recurrence rates of each subgroup were listed in Additional file 2: Table S1.

Verifying the HDL $L_{\text {Po }}$ cutoff point and its correlation with clinicopathologic characteristics in the validation cohort

The cutoff value was defined using X-tile software. To dispel possible bias from this particular cohort, we used an independent validation cohort to verify the cutoff value. As expected, Kaplan-Meier analysis showed the low- and

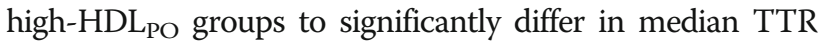
(low-HDL $L_{\mathrm{PO}}$ group: 10.0 months; high-HDL $\mathrm{HO}_{\mathrm{PO}}$ group: 
Table 2 Univariate and multivariate Cox proportional hazard analysis of factors associated with recurrence in the training cohort

\begin{tabular}{|c|c|c|c|c|}
\hline & \multicolumn{2}{|l|}{ Univariate analysis } & \multicolumn{2}{|l|}{ Multivariate analysis } \\
\hline & $\mathrm{HR}(95 \% \mathrm{Cl})$ & $P$ value & $\mathrm{HR}(95 \% \mathrm{Cl})$ & $P$ value \\
\hline Sex & $1.130(0.601-2.124)$ & 0.705 & NA & NA \\
\hline Age & $0.940(0.612-1.446)$ & 0.780 & NA & NA \\
\hline Tumor number & $0.910(0.536-1.543)$ & 0.725 & NA & NA \\
\hline Tumor size & $1.025(0.667-1.576)$ & 0.910 & NA & NA \\
\hline Tumor encapsulation & $1.115(0.727-1.710)$ & 0.617 & NA & NA \\
\hline Satellite lesion & $1.090(0.580-2.049)$ & 0.789 & NA & NA \\
\hline Vascular invasion & $0.899(0.575-1.375)$ & 0.596 & NA & NA \\
\hline Tumor grade & 1.092(0.714-1.671) & 0.685 & NA & NA \\
\hline Child-Pugh grade & 1.785(0.824-3.865) & 0.142 & NA & NA \\
\hline BCLC stage & $0.606(0.361-1.017)$ & 0.058 & NA & NA \\
\hline AFP & $0.906(0.563-1.456)$ & 0.682 & NA & NA \\
\hline $\mathrm{ALT}$ & $0.627(0.230-1.709)$ & 0.362 & NA & NA \\
\hline GGT & $1.004(1.002-1.005)$ & 0.001 & $1.986(1.308-3.017)$ & 0.001 \\
\hline $\mathrm{HbsAg}$ & $1.612(0.780-3.333)$ & 0.198 & NA & NA \\
\hline $\mathrm{HDL}_{P O}$ & $0.526(0.322-0.858)$ & 0.010 & $0.519(0.318-0.848)$ & 0.009 \\
\hline
\end{tabular}

Note:HR is hazard ratio Abbreviation: NA,not applicable

32.2 months; $P=0.0004$ Fig. $2 \mathrm{~b})$ and $\mathrm{OS}(P=0.0141$ Fig. $2 \mathrm{~d})$. The low-HDL $\mathrm{PO}_{\mathrm{PO}}$ group also had a significantly higher recurrence rate $(68.57 \%)$ than did the high-HDL $\mathrm{HO}_{\mathrm{PO}}$ group (41.50\%).

We also compared the clinicopathologic characteristics of the validation cohort with those of the training cohort, and found that they were similar in sex, age, and other characteristics (Table 3), as was the recurrence rate ( $46.70 \%$ by the end of the follow-up; median TTR: 30.9 months; range: $1.0-38.0$ months). The low-HDL $\mathrm{PO}_{\mathrm{PO}}$ group had more advanced median tumor grades $(P=0.032)$ and a higher recurrence rate $(P=0.005)$.

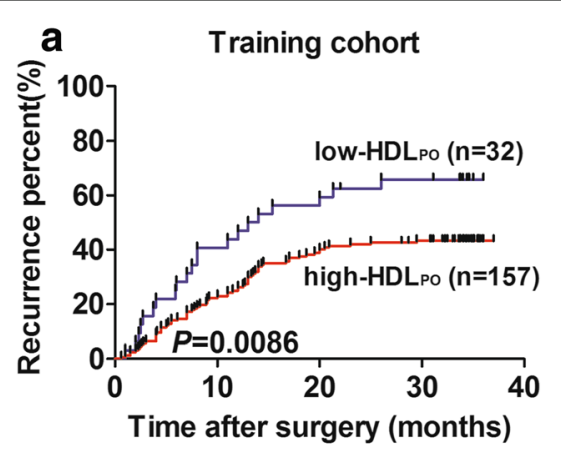

C

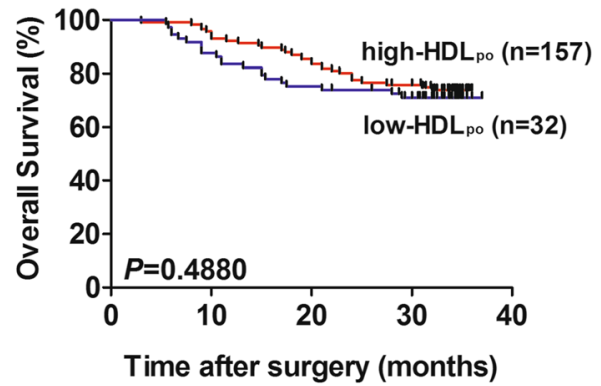

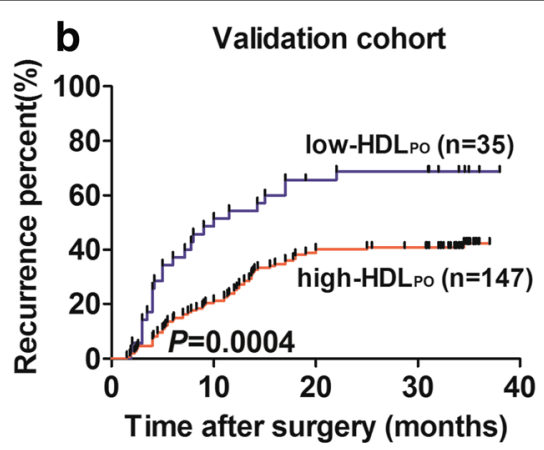

d

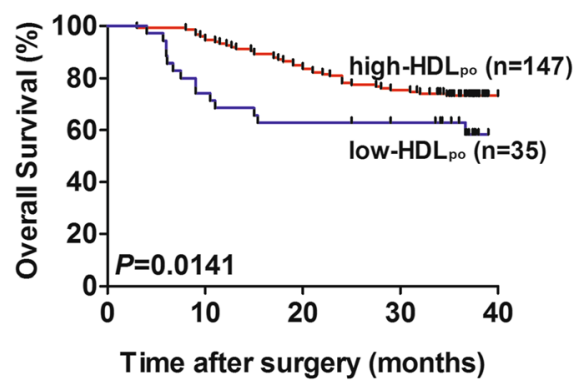

Fig. 2 Prognostic significance of HDLpo in the training and validation cohorts. Kaplan-Meier analysis for time to recurrence (TTR) and overall survival (OS) for patients with low- vs high-HDLPO in the training cohort $(\mathbf{a}, \mathbf{c})$ and the validation group (b, d) 


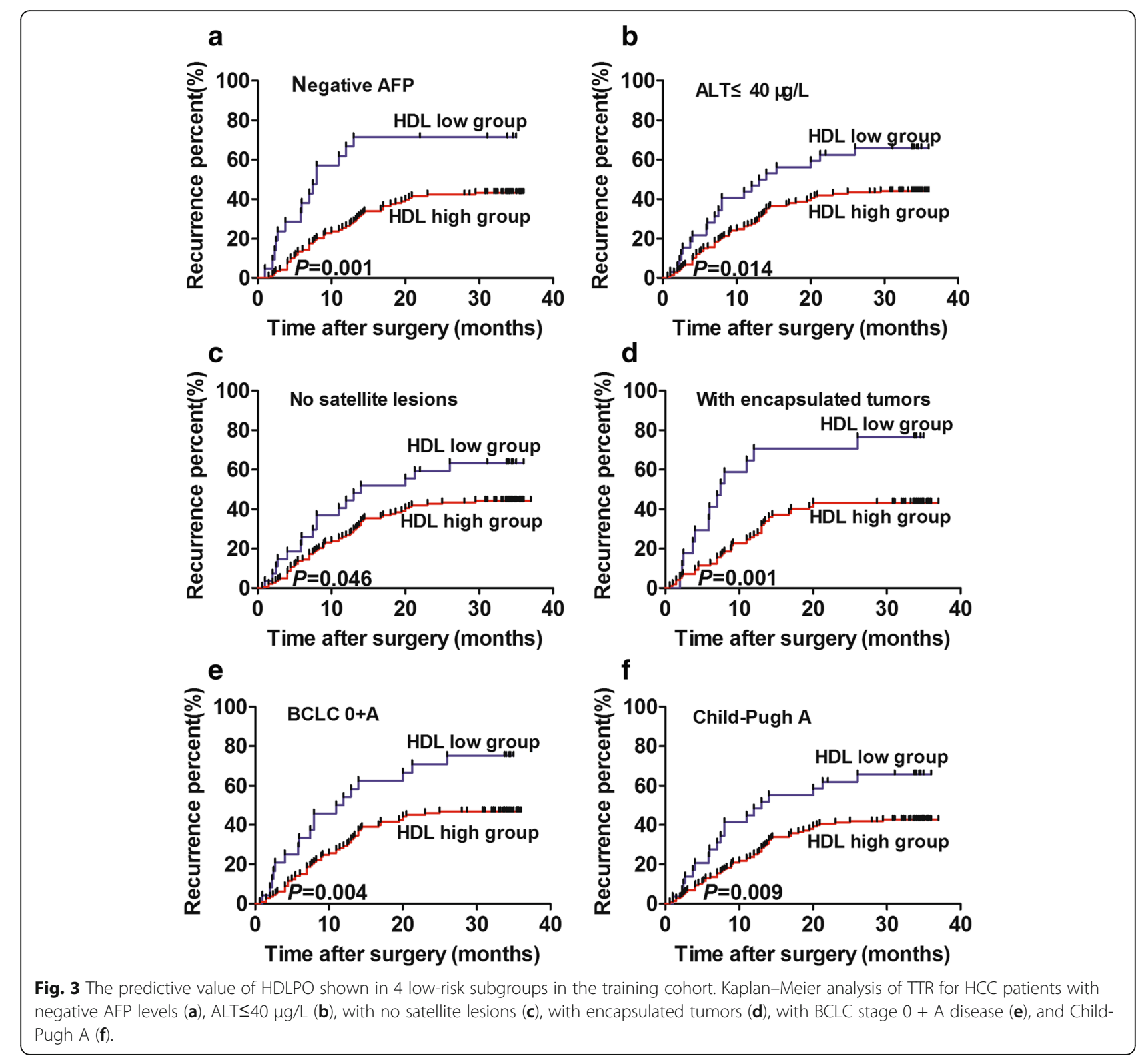

Finally, univariate analysis of the validation group showed that Child-Pugh score (HR: 2.601; 95\% CI: 1.252-5.402; $P=0.010)$, GGT (HR:1.826; 95\% CI: 1.193-2.795; $P=0.006)$ and $\mathrm{HDL}_{\mathrm{PO}}$ (HR: 0.436; 95\% CI: 0.271-0.700; $P=0.001)$ to be significantly associated with recurrence. In multivariate analysis, all of these variables independently predicted HCC recurrence (Child-Pugh score, HR: 2.358; 95\% CI: 1.125-4.945; $P=0.023$; GGT, HR: 1.863 ; 95\% CI: 1.216-2.853; $P=0.004$; and HDL $\mathrm{PO}$, HR: 0.448; 95\% CI: 0.277-0.723; $P=0.001$; Additional file 2: Table S2).

The sourse and metabolism of HDL

To explore the mechanism of low $\mathrm{HDL}_{\mathrm{PO}}$ with poor prognosis, we detected HDL mRNA level of tumor and paratumor tissues from 20 patients. The results showed that paratumor tissue presented higher level of HDL than tumor tissue $(P=0.0193$, Fig. 4a). Moreover, the cell surpernatant from high invasive HCC cell lines presented lower HDL level than normal live cell line and low invasive HCC cell lines (4b).

\section{Discussion}

A meta-analysis of 24 large randomized controlled trials of lipid-modifying therapy showed an inverse relationship between HDL and risk of cancer [14]. Fan Y, et al. demonstrated that in the triple-negative breast cancer group, patients with low HDL suffered worse relapsefree survival (RFS) and overall survival (OS), and low HDL was an independent worse prognostic factor for 
Table 3 The correlation between clinicopathologic characteristics and HDLPO in the validation cohort

\begin{tabular}{|c|c|c|c|c|c|}
\hline & & Number $(n=182)$ & Low-HDL & High-HDLpo group $(n=147)$ & $P$ value \\
\hline \multirow[t]{2}{*}{$\overline{S e x}$} & Male & 158 & 32 & 126 & $0.371^{a}$ \\
\hline & Female & 24 & 3 & 21 & \\
\hline \multirow[t]{2}{*}{ Age } & $\leq 50$ & 64 & 13 & 51 & 0.845 \\
\hline & $>50$ & 118 & 22 & 96 & \\
\hline \multirow[t]{2}{*}{ Tumor number } & Single & 144 & 27 & 117 & 0.817 \\
\hline & Multiple & 38 & 8 & 30 & \\
\hline \multirow[t]{2}{*}{ Tumor size } & $\leq 5$ & 115 & 19 & 96 & 0.246 \\
\hline & $>5$ & 67 & 16 & 51 & \\
\hline \multirow[t]{2}{*}{ Tumor encapsulation } & None & 112 & 19 & 93 & 0.340 \\
\hline & Complete & 70 & 16 & 54 & \\
\hline \multirow[t]{2}{*}{ Satellite lesions } & No & 160 & 30 & 130 & $0.663^{\mathrm{a}}$ \\
\hline & Yes & 22 & 5 & 17 & \\
\hline \multirow[t]{2}{*}{ Vascular invasion } & No & 112 & 19 & 93 & 0.340 \\
\hline & Yes & 70 & 16 & 54 & \\
\hline \multirow[t]{2}{*}{ Tumor grade } & $|-| \mid$ & 114 & 16 & 98 & 0.032 \\
\hline & III-IV & 68 & 19 & 49 & \\
\hline \multirow[t]{2}{*}{ Child-Pugh grade } & $A$ & 171 & 31 & 140 & $0.138^{\mathrm{a}}$ \\
\hline & $\mathrm{B}$ & 11 & 4 & 7 & \\
\hline \multirow[t]{2}{*}{ BCLC stage } & $0+A$ & 130 & 25 & 105 & 0.575 \\
\hline & $B+C$ & 52 & 10 & 42 & \\
\hline \multirow[t]{2}{*}{ AFP, $\mu \mathrm{g} / \mathrm{L}$} & $\leq 400$ & 135 & 22 & 113 & 0.131 \\
\hline & $>400$ & 47 & 13 & 34 & \\
\hline \multirow[t]{2}{*}{$A L T, \mu g / L$} & $\leq 40$ & 170 & 34 & 136 & $0.278^{a}$ \\
\hline & $>40$ & 12 & 1 & 11 & \\
\hline \multirow[t]{2}{*}{ GGT,IU/L } & $\leq 54$ & 101 & 19 & 82 & 1.000 \\
\hline & $>54$ & 81 & 16 & 65 & \\
\hline \multirow[t]{2}{*}{ HbsAg } & Negative & 23 & 1 & 22 & $0.053^{\mathrm{a}}$ \\
\hline & Positive & 159 & 34 & 125 & \\
\hline \multirow[t]{2}{*}{ Recurrence } & No & 97 & 11 & 86 & 0.005 \\
\hline & Yes & 85 & 24 & 61 & \\
\hline
\end{tabular}

Abbreviations: AFP, a-fetoprotein; ALT, alanine aminotransferase; GGT, Y-glutamyl transpeptadase; HBsAg, hepatitis B surface antigen; BCLC, Barcelona Clinic Liver Cancer ${ }^{\text {a}}$ Fisher's exact test

both RFS and OS [15]. Moreover, other studies had validated HDL could be used as an indicator in, lung cancer [16], prostate cancer [17], and colorectal cancer [18]. Cancer is known to be a proinflammatory state, in which inflammatory cells actively participate in the neoplastic process, allowing tumor cell proliferation, migration, and survival $[19,20]$. The relationship between inflammation and lipid metabolism has become a focus of investigation. As a metabolite, HDL plays an extremely important role in protecting the cardiovascular system from atherosclerosis, by mediating cholesterol transport. Plausibly, HDL protects against cancer development through its pleiotropic properties, including anti-oxidation and modulating cytokine production, and by blocking apoptosis, cell-growth stimulation and migration $[16,21,22]$.

Although several studies have found clues to the relationships between HDL and hepatitis or HCC [5, 23-25], we are the first to predict prognoses of HCC patients by their $\mathrm{HDL}_{\mathrm{PO}}$ levels in both training and validation groups, and to extend this predictive value to low-recurrent-risk subgroups, thereby strengthening the clinical utility of this commonly accessible test to predict HCC recurrence. As serum HDL is analyzed routinely in clinical laboratories, detection of $\mathrm{HDL}_{\mathrm{PO}}$ can easily be standardized for early decision-making to tailor the most effective therapy for each HCC patients. 

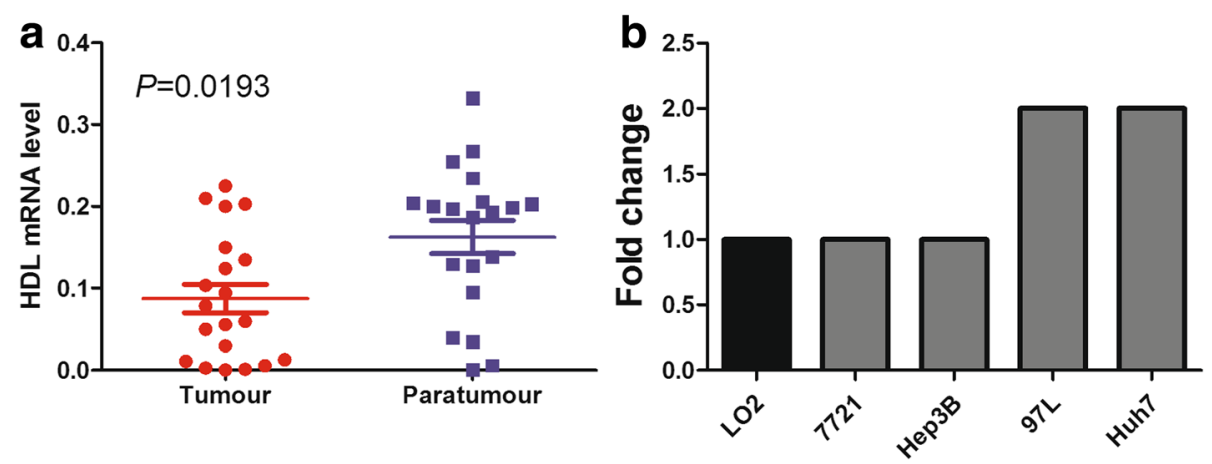

Fig. 4 The sourse and metabolism of HDL. The HDL mRNA level of tumor tissue and paratumor tissue (a); HDL concentration of HCC cell lines and normal live cell line (b)

More profoundly, we found that $\mathrm{HDL}_{\mathrm{PO}}$ was also a potent indicator for patients in four low-recurrent-risk subgroups, in whom recurrences were not easily detected by routine blood tests and pathologic results. AFP is commonly used to diagnose and monitor HCC patients' conditions after surgery [26], but its role in early dignosis and predicting prognosis of $\mathrm{HCC}$ were still in debate, especially some HCC patients were still AFP-negative, so AFP still had some limitations as an screening or predictive indicator. Satellite lesions indicate the cancer is not confined to one site and has disseminated in the liver [27]; encapsulation help limit the tumor in a separate enclosure and prevent it from spreading [28]; BCLC stage $0+\mathrm{A}$ [29] is common early HCC assessments. These factors were considered to be recurrence related, and the negative condition of them had low indices of clinical suspicion. Physicians should consider $\mathrm{HDL}_{\mathrm{PO}}$ as a stratification factor when making management decisions for HCC patients.

The progression of HCC is a complex, multi-step process, and disordered lipid metabolism might contribute to poor prognosis. It was reported that lipid oxidation and resulting oxidized lipid mediated inflammation appear to be common to the etiology of a number of inflammatory diseases [30, 31], implicating a role for lipoproteins in the development and metastasis of cancer. Since HDL was mainly formed in the liver, and our results demonstrated that HDL produced by hepatoma cells was less than normal liver cells. Jessica Fioravanti, et al. reported that after incubation of woodchuck WCH17 cell line with DiI-labled HDL, the cell line internalized the fluorescent HDL, which verified HDL could uptake by hepatoma cells [32]. HDL, as a structural component of the cell membrane and is localized in membrane microdomains that assemble the signal transduction machinery and associate to proteins implicated in key cellular signaling pathways, played the protective role from metastasis through enhancing antiinflammatory and anti-oxidant properties [33]. Because this indicator is a promising means of evaluating prognosis of patients with $\mathrm{HCC}$, we strongly recommend that doctors consider $\mathrm{HDL}_{\mathrm{PO}}$ when making management decisions. For instance, patients with lower HDL $_{\mathrm{PO}}$ should be provided with earlier and more frequent imaging or other tests to detect micro-recurrence lesions.

Our study is limited by the fact that the HDL levels were measured only once at baseline before surgery, and do not reflect random fluctuations over time, which would tend to increase the data variance. Moreover, we focused only on preoperative HDL levels without making notice of postoperative values, which confined the clinical utility of this index. As HCC patients in China have a high positive rate for HBsAg, ethnic differences in lipoprotein levels should be considered, and $\mathrm{HDL}_{\mathrm{PO}}$ cutoff values should be redefined when generalizing the findings to persons with different ethnicities.

To our knowledge, this is the first report to predict prognosis of patients with $\mathrm{HCC}$ though $\mathrm{HDL}_{\mathrm{PO}}$ levels. Although the underlying mechanism is unclear, the results validate $\mathrm{HDL}_{\mathrm{PO}}$ as an assistant predictor of post-surgical recurrence in $\mathrm{HCC}$ patients.

\section{Conclusions}

In summary, this is the first report showing a novel use of HDL in predicting the prognosis of HCC. We compared four lipid metabolites in healthy donors, chronic hepatitis patients and HCC patients and found the distinctive value of HDL. As expected, low-HDL $\mathrm{HO}_{\mathrm{PO}}$ group had a higher median tumor grade and were more likely to suffer recurrence. Moreover, the predictive value of $\mathrm{HDL}_{\mathrm{PO}}$ was retained in four low-recurrent-risk subgroups. $\mathrm{HDL}_{\mathrm{PO}}$ could be a accessible predictor of HCC recurrence after liver resections and help identify patients who need more careful monitoring and follow-up care.

\section{Additional files}

Additional file 1: Figure S1. The cutoff point generated by $\mathrm{X}$-tile. (TIFF $1880 \mathrm{~kb}$ ) 
Additional file 2: Table S1. The summary of four subgroups. Table S2. Univariate and multivariate Cox proportional hazard analysis of factors associated with recurrence in the validation cohort. (DOCX $14 \mathrm{~kb}$ )

\section{Abbreviations}

AFP: a-fetoprotein; ALT: alanine aminotransferase; BCLC: Barcelona Clinic Liver Cancer; $\mathrm{CH}$ : chronic hepatitis; GGT: -glutamyl transpeptidase; HBsAg: hepatitis virus B antigen; HCC: hepatocellular carcinoma; HD: healthy donors; HDL: highdensity lipoprotein; HDLpo: preoperative high-density lipoprotein; HR: hazard ratio; LDL: low-density lipoprotein; NA: not applicable; RCT: reverse cholesterol transport; TC: total cholesterol; TG: triglycerides; TTR: time to recurrence

\section{Acknowledgements}

The authors thank the participating patients for the source of clinical blood samples.

\section{Funding}

This study was supported by grants from the National Natural Science Foundation of China $(81,572,064,81,472,676$, and $81,572,823)$, the Nationa High Technology Research and Development Program (863 Program) of China (2015AA020401), the State Key Program of National Natural Science of China (81530077), the Projects from the Shanghai Science and Technology Commission (13,140,901,900,134119a1201, 14DZ1940300, 14,411,970,200 and $14,140,902,301)$, and the Strategic Priority Research Program of the Chinese Academy of Sciences (XDA12010202), Specialized Research Fund for the Doctoral Program of Higher Education and Research Grants Council Earmarked Research Grants Joint Research Scheme (20130071140008), Key Developing Disciplines of Shanghai Municipal Commission of Health and Family Planning (2015ZB0201), Research Project of Shanghai Municipal Commission of Health and Family Planning (201540052), The funding plan for outstanding youth doctors training, Shanghai (2016-05).

\section{Availability of data and materials}

Not applicable because of the privacy of patients.

\section{Authors' contributions}

$L T$, OY and XHG conducted the test of patients. JW, XLM, OD, CYZ performed statistical analyses. LT, YZ, YCZ wrote the manuscript. WG, XRY, BSP, JZ, JF conceived the study and participated in its design. All authors read and approved the final manuscript.

\section{Competing interests}

The authors declare that they have no conflicts of interest with the contents of this article.

\section{Ethics approval and consent to participate}

The Zhongshan Hospital Research Ethics Committee approved this study, and all patients granted written informed consent.

\section{Publisher's Note}

Springer Nature remains neutral with regard to jurisdictional claims in published maps and institutional affiliations

\section{Received: 14 February 2017 Accepted: 1 June 2017}

\section{Published online: 26 June 2017}

\section{References}

1. Forner A, Llovet JM, Bruix J. Hepatocellular carcinoma. Lancet. 2012; 379(9822):1245-55.

2. Siegel R, Naishadham D, Jemal A. Cancer statistics. CA Cancer J Clin. 2012; 62(1):10-29.

3. Utsunomiya T, Shimada M, Kudo M, et al. A comparison of the surgical outcomes among patients with HBV-positive, HCV-positive, and non-B non-C Hepatocellular carcinoma: a Nationwide study of 11,950 patients. Ann Surg. 2015;261(3):513-20.

4. Yao M, Zhao J, Lu F. Alpha-fetoprotein still is a valuable diagnostic and prognosis predicting biomarker in hepatitis B virus infection-related hepatocellular carcinoma. Oncotarget. 2016;7(4):3702-8.

5. Zhao J, Zhao Y, Wang H, Gu X, Ji J, Gao C. Association between metabolic abnormalities and HBV related hepatocelluar carcinoma in Chinese: a crosssectional study. Nutr J. 2011;10:49.
6. Bell AW. Lipid metabolism in liver and selected tissues and in the whole body of ruminant animals. Prog Lipid Res. 1979;18(3):117-64.

7. Chen YL, Peng HC, Wang XD, Yang SC. Dietary saturated fatty acids reduce hepatic lipid accumulation but induce fibrotic change in alcohol-fed rats. Hepatobiliary Surg Nutr. 2015 Jun;4(3):172-83.

8. Kontush A, Lhomme M, Chapman MJ. Unraveling the complexities of the HDL lipidome. J Lipid Res. 2013;54(11):2950-63.

9. Seko $Y$, Akuta $N$, Suzuki F, et al. Amino acid substitutions in the hepatitis $C$ virus Core region and lipid metabolism are associated with Hepatocarcinogenesis in Nonresponders to interferon plus Ribavirin combination therapy. Intervirology. 2013;56(1):13-21.

10. Sun $Y F, X u Y$, Yang $X R$, et al. Circulating stem cell - like epithelial cell adhesion molecule - positive tumor cells indicate poor prognosis of Hepatocellular carcinoma after curative resection. Hepatology. 2013;57(4):1458-68.

11. Bruix J, Llovet JM. Prognostic prediction and treatment strategy in hepatocellular carcinoma. Hepatology. 2002;35(3):519-24.

12. Yang XR, Xu Y, Shi GM, et al. Cytokeratin 10 and Cytokeratin 19: predictive markers for poor prognosis in Hepatocellular carcinoma patients after curative resection. Clin Cancer Res. 2008;14(12):3850-9.

13. Camp RL, Dolled-Filhart M, Rimm DL. X-tile: a new bio-informatics tool for biomarker assessment and outcome-based cut-point optimization. Clin Cancer Res. 2004;10(21):7252-9.

14. Jafri $\mathrm{H}$, Alsheikh-Ali AA, Karas RH. Baseline and on-treatment highdensity lipoprotein cholesterol and the risk of cancer in randomized controlled trials of lipid-altering therapy. J Am Coll Cardiol. 2010;55(25):2846-54.

15. Fan $Y$, Ding $X$, Wang J, et al. Decreased serum HDL at initial diagnosis correlates with worse outcomes for triple-negative breast cancer but not non-TNBCS. Int J Biol Markers. 2015;30(2):e200-7.

16. Kucharska-Newton AM, Rosamond WD, Schroeder JC, et al. HDL-cholesterol and the incidence of lung cancer in the atherosclerosis risk in communities (ARIC) study. Lung Cancer. 2008;61(3):292-300.

17. Mondul AM, Weinstein SJ, Virtamo J, Albanes D. Serum total and HDL cholesterol and risk of prostate cancer. Cancer Causes Control. 2011;22(11):1545-52

18. Liao F, He W, Jiang C, et al. A high LDL-Cto HDL-Cratio predicts poor prognosis for initially metastatic colorectal cancer patients with elevations in LDL-C. Onco Targets Ther. 2015;8:3135-42.

19. Coussens LM, Werb Z. Inflammation and cancer. Nature. 2002:420(6917):860-7.

20. Gonda TA, Tu S, Wang TC. Chronic inflammation, the tumor microenvironment and carcinogenesis. Cell Cycle. 2009;8(13):2005-13.

21. Ramadori P, Kroy D, Streetz KL. Immunoregulation by lipids during the development of non-alcoholic steatohepatitis. Hepatobiliary Surg Nutr. 2015;4(1):11-23.

22. Inoue $M$, Kurahashi $N$, Iwasaki $M$, et al. Metabolic factors and subsequent risk of hepatocellular carcinoma by hepatitis virus infection status: a large-scale population-based cohort study of Japanese men and women (JPHC study cohort II). Cancer Causes Control. 2009:20(5):741-50

23. Khattab MA, Eslam M, Mousa YI, et al. Association between metabolic abnormalities and hepatitis C-related hepatocellular carcinoma. Ann Hepatol. 2012;11(4):487-94.

24. Kim YK, Zuccaro MV, Zhang C, Sarkar D, Quadro L. Alcohol exposure in utero perturbs retinoid homeostasis in adult rats. Hepatobiliary Surg Nutr. 2015;4(4):268-77.

25. Jiang J, Nilsson-Ehle P, Xu N. Influence of liver cancer on lipid and lipoprotein metabolism. Lipids Health Dis. 2006;5:4.

26. Xu WJ, Guo BL, Han YG, Shi L, Ma WS. Diagnostic value of alphafetoprotein-L3 and Golgi protein 73 in hepatocellular carcinomas with lowAFP levels. Tumour Biol. 2014;35(12):12069-74.

27. Thng Y, Tan JK, Shridhar IG, Chang SK, Madhavan K, Kow AW. Outcomes of resection of giant hepatocellular carcinoma in a tertiary institution: does size matter? HPB (Oxford). 2015;17(11):988-93.

28. Wu CC, Shen $\mathrm{CH}$, Liu HT, et al. Unroofing hepatectomy: a facilitating approach for resection of deep-seated hepatocellular carcinoma adjacent to major intrahepatic vessels in cirrhotic patients. J Surg Oncol. 2015;111(4):396-403.

29. Richani M, Kolly P, Knoepfli M, et al. Treatment allocation in hepatocellular carcinoma: assessment of the BCLC algorithm. Ann Hepatol. 2016;15(1):82-90.

30. Bochkov VN, Oskolkova OV, Birukov KG, et al. Generation and biological activities of oxidized phospholipids. Antioxid Redox Signal. 2010;12:1009-59. 
31. Wang L, Gill R, Pedersen $T L$, et al. Triglyceride-rich lipoprotein lipolysis releases neutral and oxidized FFAs that induce endothelial cell inflammation. J Lipid Res. 2009:50:204-13.

32. Mamidi S, Höne S, Kirschfink M. The complement system in cancer: ambivalence between tumour destruction and promotion. Immunobiology. 2017;222(1):45-54.

33. Zhang X, Wang L, Chen B. Recombinant HDL (Milano) protects endotoxinchallenged rats from multiple organ injury and dysfunction. Bio Chem. 2015;396(1):53-60

Submit your next manuscript to BioMed Central and we will help you at every step:

- We accept pre-submission inquiries

- Our selector tool helps you to find the most relevant journal

- We provide round the clock customer support

- Convenient online submission

- Thorough peer review

- Inclusion in PubMed and all major indexing services

- Maximum visibility for your research

Submit your manuscript at www.biomedcentral.com/submit 